\title{
Editorial
}

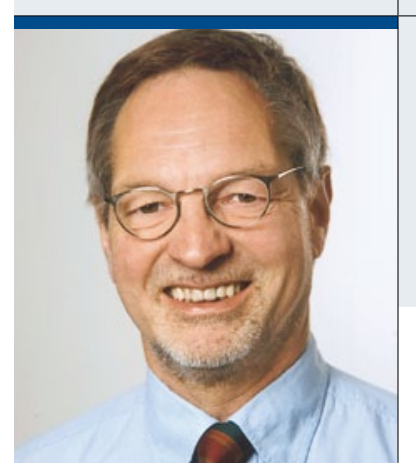

"Die mikroskopische Harnuntersuchung kann

nur noch mit Subventionierung durch den

behandelnden Arzt erbracht werden."

Dr. med. Walther Grohmann

Chefredakteur und niedergelassener Urologe, München

\section{Kollegen, protestiert!}

D as altehrwürdige, kreisrunde DGU-Symbol, der Urologe mit dem Harnschauglas, wird auch in diesem Jahr auf dem DGU-Kongress in Dresden allerorts zu sehen sein. Seit dem 1. Juli dieses Jahres werden jedoch mit dem Wort „Harnschau“ Emotionen, ja Wut verbunden: Seit diesem Zeitpunkt gilt laut der aktuellen Richtlinie der Bundesärztekammer für die Beurteilung des Urinsediments die Bestimmung, dass eine externe Qualitätssicherung durch einen Ringversuch verpflichtend ist. Kuriosität am Rande: Den Begriff "Urinsediment" gibt es im EBM nicht, dort ist von der "Harnmikroskopie“ die Rede. In der GOÄ ist der Begriff "Urinsediment" dagegen ausgesprochen - und somit hat hier die grundsätzliche Ringversuchspflicht wie von der Bundesärztekammer angeordnet (auch) für Privatpatienten sogar verbal korrekt Gültigkeit. Weiteres zu den Fakten finden Sie im Interview auf Seite 20.

Dass die EBM-Honorierung der mikroskopischen Harnuntersuchung im Cent-Bereich liegt und dem neben dem Aufwand für die Untersuchung nun auch noch die Kosten für die Qualitätssicherung mit den damit verbundenen Umständen gegenüberstehen, lässt nur einen Schluss zu: Diese urologische Basisdiagnostik kann nur noch mit Subventionierung durch den behandelnden Arzt erbracht werden. Gynäkologen, die dazu verpflichtet sind, das Urinsediment im Rahmen der Betreuung von Schwangeren zu untersuchen, stehen vor demselben Problem. Sie diskutieren, den Urin zur Untersuchung in das Gemeinschaftslabor zu schicken, um finanzielle Verluste zu vermeiden, wobei die Problematik der „Selbsterbringung der Leistung" mit eingeschlossen ist. Neben der ausbleibenden sofortigen Verfügbarkeit des Untersuchungsergebnisses bleibt aber unter anderem die Frage, ob Trichomonaden inzwischen zu einer Zeit- und Versand-resistenten Spezies mutiert sind.

\section{Farbe bekennen!}

Mit diesem Heft startet URO-NEWS eine Serie, in der wir Sie nach Ihrer Meinung fragen. In der vorliegenden Ausgabe fragen wir Sie, wie Sie sich die Zukunft unserer Verbände wünschen. Nicht alle Urologen werden in Dresden auf dem Kongress sein und nicht alle sind Mitglieder von DGU und/oder BDU. Manche haben hierfür gute Gründe und gerade auch deren Meinung soll Gehör finden (Beitrag und zugehörige Fragen auf den Seiten $22 \mathrm{f}$.).

Die unsinnige und kontraproduktive Richtlinie zum qualitätsgesicherten Urinsediment hat uns dieses mal zum Start der Serie „Farbe bekennen“ - veranlasst, eine Zusatzfrage anzuhängen, in der Sie Ihrer Meinung hierzu Luft machen können. Offen gesagt: Bei dieser Frage rechnen wir mit Ihrem massiven Protest unter reger Beteiligung per Fax oder über unsere Internetseite. Dieser könnte dann auch für die angesprochenen Verbände als wichtige Grundlage für Verhandlungen dienen!

Ihr

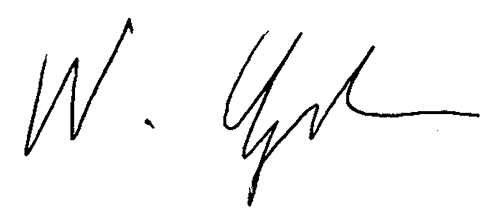

